\title{
Comunicación en el tercer sector antes y durante la Pandemia COVID 19: estrategias de comunicación en las redes sociales de las ONG de Blumenau, Santa Catarina, Brasil
}

Communication in the third sector before and during the COVID 19 pandemic. Communication strategies on social networks of NGOs in Blumenau, Santa Catarina, Brazil

Dra. Fabricia Durieux Zucco

Universidade do Vale do Itajaí e Universidade Regional de Blumenau | 5ạ Avenida, 1100 | Balneário Camboriú | Brasil | https://orcid.org/0000-0001-5538-1195 |

fabriciazucco@hotmail.com

Juliana Machado

Universidade Regional de Blumenau | R. Antônio da Veiga, 140| Blumenau | Brasil | https://orcid.org/0000-0003-1715-8270 | julianamachado.pp@gmail.com

Cynthia Morgana Boos de Quadros

Universidade Regional de Blumenau | R. Antônio da Veiga, 140| Blumenau | Brasil | https://orcid.org/0000-0001-6729-7361 | cynthiadequadros@gmail.com

Thamires Foletto Fiuza

Universidade do Vale do Itajaí | 5ạ Avenida, 1100 | Balneário Camboriú | Brasil |

https://orcid.org/0000-0002-3014-2611 | thamiresf.fiuza@gmail.com

Fechas | Recepción: 21/12/2020 | Aceptación: 28/02/2021 


\section{Resumen}

Las redes sociales se han convertido en una parte importante de la comunicación para las organizaciones sin fines de lucro al ofrecerles nuevas oportunidades para compartir, colaborar y movilizar la acción colectiva, especialmente durante la pandemia del COVID-19. El presente estudio busca identificar la presencia en línea y analizar las estrategias de comunicación utilizadas en las redes sociales de las ONG en la ciudad de Blumenau, Santa Catarina, Brasil. Desde una perspectiva metodológica mixta y adoptando un enfoque exploratorio y descriptivo, los métodos y procedimientos de investigación incluyeron una revisión bibliográfica $y$ documental, y un análisis de contenido. De esa forma, se analizaron 1.400 publicaciones de 14 organizaciones del tercer sector de Blumenau, Santa Catarina, Brasil, en las redes sociales Facebook, Instagram y Twitter, así como el compromiso online generado por los usuarios entre noviembre de 2019 y mayo de 2020, antes y durante la pandemia provocada por el COVID-19. Para tales propósitos, fueron utilizados los postulados de Deschamps y McNutt (2014) relacionados a los objetivos para el uso de las redes sociales por parte de organizaciones del tercer sector. Los resultados destacan la importancia de la presencia de las ONG en las redes sociales, fundamentalmente en tiempos de crisis. La estrategia de comunicación más utilizada fue la de involucrar a la sociedad civil. El artículo también proporciona contribuciones teóricas y de gestión.

Palabras clave: tercer sector, redes sociales, engagement, estrategias de comunicación.
Abstract

Social media has become a significant part of communication for nonprofits by offering them new opportunities to share, collaborate, and mobilize collective action, especially during the COVID-19 pandemic. This study seeks to identify the online presence and review the communication strategies used in the social networks of NGOs in the city of Blumenau, Santa Catarina, Brazil. From a mixed methodological perspective and adopting an exploratory and descriptive approach, the research methods and procedures included a bibliographic and documentary review and content analysis. In this way, 1,400 publications from 14 organizations from the third sector of Blumenau, Santa Catarina, Brazil were analyzed, on the social networks Facebook, Instagram and Twitter, as well as the online engagement generated by users between November 2019 and May 2020, before and after COVID-19. For such purposes, the postulates of Deschamps and McNutt (2014) related to the objectives for the use of social networks by third sector organizations were used. The results highlighted the importance of the presence of NGOs in social networks, mainly in times of crisis. The most used communication strategy was to involve civil society.

Keywords: third sector, social networks, engagement, communication Strategies.

\section{INTRODUCCIÓN}

En los últimos años, las Organizaciones No Gubernamentales $(\mathrm{ONG})^{1}$ conocidas como instituciones del tercer sector, han crecido tanto en número como en tamaño, con el

\footnotetext{
${ }^{1}$ El término Organización No Gubernamental (ONG), también conocido en Brasil como Organización de la Sociedad Civil (OSC), define a las entidades que simultáneamente caen bajo cinco criterios: i) son privadas y no están legalmente vinculadas al Estado; ii) no sirven para fines lucrativos; iii) están legalmente constituidas, tienen personalidad jurídica y están inscritas en el Registro Nacional de Personas Jurídicas (CNPJ); iv) se auto-administran y gestionan sus actividades de forma autónoma; y v) se constituyen voluntariamente (IBGE, 2019).
} 
compromiso de suplir la ausencia del Estado en algunos servicios de necesidades sociales (Silva, 2018). Según un estudio del Instituto de Investigaciones Económicas Aplicadas (IPEA), institución vinculada al Gobierno Federal, en 2017 Brasil cerró el año con 820 mil ONG existentes², lo que frente a la última cifra disponible, 400, es un aumento muy considerable. En cuanto a la presencia regional, la región Sudeste presentó el $40 \%$ de las organizaciones, seguida de Noreste (25\%), Sur (19\%), Medio Oeste (8\%) y Región Norte (8\%) (Mello et al., 2019).

Por lo tanto, las ONG necesitan buscar apoyo financiero de otras organizaciones, ya sea del sector privado, público o de particulares. Ante esto, es posible comprender la importancia de que las organizaciones utilicen el potencial que ofrece la comunicación como aliado en la defensa de sus intereses, ya sea para obtener recursos, reclutar voluntarios y simpatizantes, incrementar el compromiso o rendir cuentas a la sociedad.

Aunque las organizaciones sin fines de lucro son diferentes de aquellas orientadas al mercado, algunas estrategias y herramientas de comunicación utilizadas pueden implementarse para satisfacer las necesidades de las ONG sin negar su verdadera misión. Ya sea a través de estrategias que identifiquen al público más interesado en apoyar la causa (segmentación de mercado) o hacer más atractiva la comunicación para estas personas, utilizando los canales más adecuados (Dolnicar y Lazarevski, 2009).

Según Moreira (2014), debido a la falta de recursos económicos y la restricción de espacio que ponen a disposición los medios tradicionales, las organizaciones del tercer sector deben buscar medios alternativos según su contexto y disponibilidad. Así como el sector empresarial comenzó a utilizar Internet para infiltrarse en nuevos mercados y relacionarse con sus consumidores, las organizaciones sin fines de lucro también están viendo las nuevas oportunidades que han surgido de las herramientas digitales para acciones de participación y ciudadanía.

Internet juega un papel muy importante en la configuración de la imagen de las organizaciones del tercer sector, ya que les permite acercarse a sus diversos públicos. Las ONG utilizan cada vez más la comunicación en diferentes plataformas de Internet para recaudar fondos, mejorar las relaciones, aumentar sus esfuerzos de movilización y mantener a sus audiencias bien informadas. Sin embargo, no todas las organizaciones sin fines de lucro están aprovechando al máximo el potencial en línea (Pope et al. 2009).

Las redes sociales ofrecen a las organizaciones oportunidades para relacionarse e involucrar a los miembros existentes y potenciales partidarios con nuevas oportunidades para compartir, colaborar y movilizar la acción colectiva (Greenberg y Macaulay, 2009). Ellas se han convertido en una parte importante de la comunicación para las organizaciones sin fines de lucro. El uso de los recursos de las redes sociales para el tercer sector ha sido estudiado por varios investigadores con el fin de comprender cómo estas organizaciones pueden aprovechar tal servicio (Campbell y Lambright, 2019; Deschamps y McNutt, 2014; Lovejoy y Saxton, 2012). A pesar de esto, las ONG enfrentan varios desafíos al desarrollar una estrategia de redes sociales.

\footnotetext{
2 Últimos datos publicados por el IPEA e identificados por el Registro Nacional de Identificación Social (CNIS), base de datos del gobierno federal brasilero que almacena información laboral y de seguridad social. Los datos de registro de los formularios del CNPJ son la principal referencia para la identificación de entidades privadas y sin fines de lucro en Brasil, permitiendo el mapeo de las ONGs (Mello et al., 2019).
} 
Según Tian (2019), el principal es la falta de recursos, generalmente en términos de personas y tiempo para planificar y ejecutar la estrategia.

En el contexto actual las ONG enfrentan otro desafío. A raíz de eventos recientes y sin precedentes relacionados con la pandemia del COVID-19, las organizaciones sin fines de lucro están bajo presión para brindar servicios valiosos mientras operan en circunstancias increíblemente desafiantes. Las prácticas profesionales de las ONG se han alterado y las consecuencias van desde la cancelación de la programación y los eventos habituales, el aumento de las necesidades de servicios de primera línea, las interrupciones de la financiación, la conversión al trabajo remoto, así como la adopción de nuevos protocolos operativos. Las redes sociales han servido, de cierta forma, para involucrar a la comunidad y desarrollar nuevas asociaciones en medio de esta pandemia.

Atendiendo a tales criterios, este estudio pretende identificar la presencia en línea y revisar las estrategias de comunicación utilizadas en las redes sociales de las ONG en la ciudad de Blumenau, Santa Catarina, Brasil. Así, se busca analizar el engagement (compromiso) online generado por los usuarios en las redes sociales estudiadas (Facebook, Instagram y Twitter) entre noviembre de 2019 y mayo de 2020, antes y durante la pandemia provocada por el COVID-19. Para tales propósitos, fueron utilizados los postulados de Deschamps y McNutt (2014) relacionados a los objetivos para el uso de las redes sociales por parte de organizaciones del tercer sector: 1) comunicarse con los beneficiarios, 2) atraer voluntarios y donaciones, 3) involucrar a la sociedad civil y 4) mejorar la rendición de cuentas y transparencia.

\section{MARCO TEÓRICO}

\subsection{Comunicación y marketing en el tercer sector}

Aunque muchas organizaciones sin fines de lucro pueden ver el marketing como una actividad comercial, Kotler y Levy (1969) creen que juega un papel muy importante en la vida de las organizaciones sin fines de lucro y todas las organizaciones realizan marketing, lo sepan o no. Además, el sector sin fines de lucro tiene características similares a los problemas del mercado, donde las asociaciones disminuyen, los costos aumentan y la competencia es feroz.

Según Pope et al. (2009), las técnicas de marketing para ONG no se aplicaron hasta la década de 1970, sin embargo, hoy en día es una práctica bien aceptada. Estas se consideran necesarias para la captación de fondos, que es un gran agravante en el tercer sector, ya que pocas inversiones se destinan a acciones sociales y empresas y particulares no están tan interesados en contribuir a las causas sociales (Silva, 2018). Debido a estos indicadores, se sugirió que el marketing tenía mucho que ofrecer al tercer sector.

El marketing social es una disciplina de marketing distinta, etiquetada como tal desde principios de la década de 1970, y se refiere principalmente a los esfuerzos centrados en influir en los comportamientos que contribuyen a las comunidades. Utiliza principios y técnicas de marketing para crear, comunicar y agregar valor en beneficio de la sociedad. En el sector sin fines de lucro, el marketing se utiliza para respaldar los servicios de la organización, la compra de productos y servicios auxiliares, la contratación de voluntarios, los esfuerzos de promoción y la recaudación de fondos (Lee y Kotler, 2008).

Según Morales (2016) la principal diferencia entre el marketing tradicional y el marketing social es su propia naturaleza. En otras palabras, sus acciones están dirigidas a la sociedad y con el fin 
de solucionar problemas de carácter colectivo y el bienestar de los ciudadanos. Su función principal es contribuir a la difusión de causas e ideas sociales. En el marketing social, no sólo es necesario estimular los cambios sociales (mercado de consumo), sino también trabajar con personas que desarrollan acciones convincentes (organización de mercado) y con quienes brindan apoyo financiero y material para financiar las actividades sociales propuestas (mercado patrocinador) (Morales, 2016).

Peruzzo (2013) señala que las comunicaciones en ONG, Asociaciones Comunitarias y Movimientos Sociales ocurren en dos niveles diferentes: 1) comunicación movilizadora, dirigida a los beneficiarios, es decir, al sujeto directo involucrado en las acciones de la institución; y 2) comunicación institucional, dirigida a otros públicos y al conjunto de la sociedad. Para que una ONG pueda obtener más recursos es necesario contar con una estrategia de comunicación dirigida a los posibles inversores sobre las acciones sociales a las que apunta, y al mismo tiempo, demostrar claridad sobre el objetivo del proyecto, lo que quiere lograr, los ideales y la misión de la institución, así como su visión y valores bien definidos. Si la comunicación no se ejecuta bien, sin la debida planificación y mano de obra calificada, puede resultar en estancamientos con activistas, voluntarios, donantes o posibles donantes (Silva, 2018). En este sentido, resulta de extrema importancia contar con una planificación estratégica de comunicación (Esparcia et al., 2017; Arroyo-Almaraz y Calle Mendoza, 2018; González et al., 2018; Román-San-Miguel y Díaz, 2019).

Para Machado y Almeida (2015), además de brindar un clima propicio para las donaciones, la comunicación debe realizarse de manera que favorezca las relaciones duraderas y aumente la conciencia de estos donantes y potenciales voluntarios sobre la organización. Mediante materiales publicitarios como folletos, libros, boletines informativos y mediante asociaciones con oficinas de prensa, por ejemplo, las ONG pueden ganar más espacio y fortalecer los esfuerzos de recaudación de fondos.

\section{METOdOLOGÍA}

En el presente trabajo, se buscó identificar la presencia en línea y revisar las estrategias de comunicación utilizadas de las ONG en la ciudad de Blumenau, Santa Catarina, Brasil, así como analizar el engagement (compromiso) online generado por los usuarios en las redes sociales estudiadas (Facebook, Instagram y Twitter). Dado este criterio, desde una perspectiva metodológica mixta y adoptando un enfoque exploratorio y descriptivo, los métodos y procedimientos de investigación incluyeron una revisión bibliográfica y documental, y un análisis de contenido. Según Bardin (2006), este último es un conjunto de técnicas de análisis de comunicaciones, que utiliza procedimientos sistemáticos y objetivos para la descripción de mensajes, en los que se busca describir el proceso de comunicación, ya sea a través de discursos o textos.

La selección de la muestra fue no aleatoria (Mattar, 2001), por lo que los elementos de la población se escogieron deliberadamente en función del juicio de los investigadores. Se consideró como población a catorce Organizaciones No Gubernamentales (ONG) que viven, de cierta manera, la misma realidad, y están registradas ante la CMDCA (Consejo Municipal de los Derechos de la Niñez y la Adolescencia) de Blumenau-SC. Ellas son: Centro de Integración Empresa-Escuela - CIEE; Centro de Recuperación Nova Esperança - CERENE; Asociación de Educación Complementaria - Puro Amor; Asociación de Padres y Amigos de Personas 
Excepcionales - APAE; Polo Tecnológico de Información y Comunicación de la Región de Blumenau - BLUSOFT; Asociación Casa de Apoyo; Sociedad Casa da Esperanza; Asociación Asistencial Lar Betânia; Asociación de Voluntarios de São Roque; Asociación Blumenauense de Amigos de Personas con Discapacidad Auditiva - ABADA; Asociación Casa de Acogida São Feli-pe Neri; Asociación Child First; Centro Provisional de Servicios Socioeducativos Blumenau - CASEP y Generación de Empleo y Renta y Apoyo al Desarrollo Regional - GERAR. Además, se descartaron seis ONG registradas ante la CMDCA por dos motivos: 1) tener una página con cobertura nacional; 2) no poseer o estar activo en las redes sociales elegidas en el período estipulado para el análisis de datos.

La recogida de datos se realizó a través de Internet, con la finalidad de analizar el engagement de los usuarios en las redes sociales de las ONG elegidas, a través de sus publicaciones, en los medios de Facebook, Instagram y Twitter, que se encuentran entre las diez redes sociales más utilizadas por brasileños, según una encuesta realizada por We are Social - Agencia creativa global socialmente dirigida en 2019 y publicada en la página de Resultados Digitales.

Para el análisis de datos se consideraron cuatro objetivos para el uso de las redes sociales por parte de organizaciones del tercer sector, según los autores Deschamps y McNutt (2014): 1) comunicarse con los beneficiarios (representado como "comunicación"); 2) captar voluntarios y donaciones (representado como "solidario"); 3) involucrar a la sociedad civil (representado como "institucional"); y 4) mejorar la responsabilidad y la transparencia (representado como "transparencia"). A pesar de tener cuatro objetivos distintos, se consideró la posibilidad de identificar diferentes objetivos en una sola publicación. Por lo tanto, mientras la ONG se comunica con los beneficiarios, puede involucrar a la sociedad civil.

El período completo de recopilación de datos fue de seis meses, desde el 24/11/19 hasta el 24/5/20. Sin embargo, este período se dividió en dos partes: $24 / 11 / 19$ al 25/2/20 que caracteriza antes del inicio de la pandemia del virus COVID-19 en Brasil y 26/02/20 al 24/05/2020 después de la propagación del virus en territorio brasileño. El objetivo fue verificar si hubo cambios en la forma en que las ONG se comunicaron con respecto al período anterior.

La recolección de datos de participación de ONG en redes sociales fue cuantificada a través de un formulario de categorización para cada red social, en el período de 6 días, del 24 al 30 de mayo de 2020. El formulario de categorización incluyó los siguientes aspectos de análisis: a) Fecha de publicación; b) Identificación de la organización; c) Objetivo de comunicación, teniendo en cuenta los cuatro aspectos descritos por Deschamps y McNutt (2014); d) Cantidad de reacciones que son: Me gusta, Amado, Triste, Guau, Grr, Fuerza, Jaja; e) Número de comentarios, ya sean positivos, neutrales o negativos; f) Número de acciones.

\section{RESULTADOS Y DISCUSIÓN}

\subsection{Resultados generales}

Durante el período del 24/11/19 al 24/05/20 se analizaron 1.400 publicaciones de 14 organizaciones del tercer sector de Blumenau, en las redes sociales Facebook, Instagram y Twitter. De ellas, 837 publicaciones se realizaron en Facebook, 520 en Instagram y 43 en Twitter. Estas publicaciones generaron 58.874 interacciones en total, en todas las redes sociales. Las interacciones se contabilizan considerando el compromiso de los usuarios a través de reacciones 
(like, amado, triste, fuerza, grr y jaja), comentarios (positivos, neutrales y negativos) y compartidos.

Para el análisis de contenido abordado por las organizaciones, se utilizaron los cuatro objetivos que según Deschamps y McNutt (2014) ayudan a las organizaciones sin fines de lucro a organizar, planificar y monitorear actividades en las redes sociales. En todas las páginas de redes sociales se contabilizó el número de publicaciones de cada ONG, de acuerdo con los objetivos mencionados.

Tabla 1

Publicación por objetivo en Facebook

\begin{tabular}{|c|l|c|c|c|c|c|}
\hline \multicolumn{1}{|c|}{ ONG } & OBJETIVO 1 & OBJETIVO 2 & OBJETIVO 3 & OBJETIVO 4 & No. POSTS \\
\hline $\mathbf{1}$ & SÃO ROQUE & 9 & 12 & 146 & 11 & 162 \\
\hline $\mathbf{2}$ & APAE & 94 & 5 & 80 & 14 & 1469 \\
\hline $\mathbf{3}$ & CERENE & 9 & 21 & 74 & 62 & 115 \\
\hline $\mathbf{4}$ & CIEE & 84 & 9 & 65 & 2 & 111 \\
\hline $\mathbf{5}$ & BLUSOFT & 33 & 4 & 50 & 18 & 59 \\
\hline $\mathbf{6}$ & PURO AMOR & 6 & 3 & 42 & 17 & 48 \\
\hline $\mathbf{7}$ & CRIANÇA EM PRIMEIRO LUGAR & 13 & 1 & 33 & 8 & 39 \\
\hline $\mathbf{8}$ & SÃO FELIPE NERI & 1 & 16 & 17 & 11 & 38 \\
\hline $\mathbf{9}$ & CASA DE APOIO & 2 & 14 & 21 & 2 & 36 \\
\hline $\mathbf{1 0}$ & GERAR & 19 & 0 & 16 & 3 & 31 \\
\hline $\mathbf{1 1}$ & CASA DA ESPERANÇA & 2 & 9 & 13 & 5 & 23 \\
\hline $\mathbf{1 2}$ & ABADA & 15 & 2 & 11 & 2 & 18 \\
\hline $\mathbf{1 3}$ & LAR BETANIA & 4 & 0 & 4 & 4 & 7 \\
\hline $\mathbf{1 4}$ & CASEP & 0 & 0 & 3 & 1 & 4 \\
\hline \multicolumn{2}{|l|}{ TOTAL GENERAL } & $\mathbf{2 9 1}$ & $\mathbf{9 6}$ & $\mathbf{5 7 5}$ & $\mathbf{1 6 0}$ & $\mathbf{8 3 7}$ \\
\hline
\end{tabular}

Fuente: elaboración propia.

En la red social Facebook se observaron un total de 291 publicaciones relacionadas con el objetivo 1 (comunicación), 96 con el objetivo 2 (solidario), 575 con el objetivo 3 (institucional), y 160 con el objetivo 4 (transparencia). En Facebook, el objetivo más explorado fue el 3, involucrar a la sociedad civil, que según Deschamps y McNutt (2014) utilizan las redes sociales para movilizar a las masas y promover temas de sensibilización y conectar a las personas con las causas defendidas.

A diferencia de la red social Facebook, no todas las ONG están presentes y activas en Instagram, de las 14 organizaciones analizadas, 13 estuvieron activas en las redes dentro del período analizado, sin embargo, cabe mencionar que si bien el número de ONG es menor, el número de publicaciones aún es más pequeño en comparación con Facebook.

Tabla 2

Publicación por objetivo en Instagram

\begin{tabular}{|c|c|c|c|c|c|c|}
\hline & ONG & OBJETIVO 1 & OBJETIVO 2 & OBJETIVO 3 & OBJETIVO 4 & No. POSTS \\
\hline 1 & APAE & 71 & 4 & 37 & 5 & 100 \\
\hline 2 & SÃO ROQUE & 14 & 4 & 79 & 2 & 95 \\
\hline 3 & CERENE & 4 & 11 & 75 & 30 & 87 \\
\hline 4 & BLUSOFT & 32 & 4 & 45 & 8 & 56 \\
\hline 5 & PURO AMOR & 8 & 4 & 41 & 10 & 47 \\
\hline 6 & SÃO FELIPE NERI & 1 & 16 & 11 & 4 & 38 \\
\hline 7 & CASA DE APOIO & 0 & 16 & 13 & 1 & 29 \\
\hline 8 & CRIANÇA EM PRIMEIRO LUGAR & 7 & 0 & 10 & 3 & 15 \\
\hline 9 & LAR BETANIA & 2 & 1 & 11 & 1 & 13 \\
\hline 10 & ABADA & 5 & 3 & 8 & 2 & 13 \\
\hline 11 & CASA DA ESPERANÇA & 0 & 3 & 7 & 2 & 10 \\
\hline 12 & GERAR & 5 & 0 & 6 & 1 & 10 \\
\hline 13 & CIEE & 3 & 2 & 2 & 2 & 7 \\
\hline
\end{tabular}




\begin{tabular}{|c|c|c|c|c|c|}
\hline TOTAL GENERAL & $\mathbf{1 5 2}$ & $\mathbf{6 8}$ & $\mathbf{3 4 5}$ & $\mathbf{7 1}$ & $\mathbf{5 2 0}$ \\
\hline
\end{tabular}

Se observó que la mayoría de las publicaciones realizadas en Facebook fueron replicadas en Instagram, por lo que el objetivo 3 (institucional) también se destacó en esta red social, seguido del objetivo 1 (comunicación), 4 (transparencia) y finalmente el objetivo 2 (solidario).

La red social Twitter fue poco explotada por las organizaciones. Sólo una ONG utiliza esta red social, CIEE - Centro de Integração Empresa-Escola, que tiene comunicación dirigida a jóvenes que quieren encontrar vacantes para pasantías o aprendices. Al igual que Instagram, las publicaciones de Facebook también se replicaron en Twitter y el objetivo tres fue el más utilizado.

Tabla 3

Publicación por objetivo enTwitter

\begin{tabular}{|c|c|c|c|c|c|c|}
\hline & ONG & OBJETIVO 1 & OBJETIVO 2 & OBJETIVO 3 & OBJETIVO 4 & No. POSTS \\
\hline $\mathbf{1}$ & CIEE & 26 & 5 & 33 & 1 & 43 \\
\hline \multicolumn{2}{|c|}{ TOTAL GENERAL } & $\mathbf{2 6}$ & $\mathbf{5}$ & $\mathbf{3 3}$ & $\mathbf{1}$ & $\mathbf{4 3}$ \\
\hline
\end{tabular}

Fuente: elaboración propia.

La siguiente tabla muestra el número de publicaciones según los cuatro objetivos, considerando las tres redes sociales, Facebook, Instagram y Twitter. El objetivo 3 (institucional) es el más utilizado por las organizaciones con 953 publicaciones, justo después del objetivo 1 (comunicación) con 469 publicaciones, seguido del objeto 4 (transparencia) con 232 publicaciones y finalmente el objetivo 2 (solidaridad), con 169 publicaciones, respectivamente.

Tabla 4

Resumen de publicaciones por objetivo

\begin{tabular}{|c|c|c|c|c|c|}
\hline & REDE SOCIAL & OBJETIVO 1 & OBJETIVO 2 & OBJETIVO 3 & OBJETIVO 4 \\
\hline 1 & FACEBOOK & 291 & 96 & 575 & 160 \\
\hline 2 & INSTAGRAM & 152 & 68 & 345 & 71 \\
\hline 3 & TWITTER & 26 & 5 & 33 & 1 \\
\hline \multicolumn{2}{|r|}{ TOTAL GENERAL } & 469 & 169 & 953 & 232 \\
\hline
\end{tabular}

Fuente: elaboración propia.

\subsection{Antes y durante la pandemia provocada por el COVID-19}

Para comprender el fenómeno de la comunicación de las ONG antes y durante la pandemia del COVID-19, se realizó un estudio comparativo entre los períodos en que el virus aún no había llegado a Brasil (24/11/19 - 25/02/20) y el período en el que se extendió en el país, a partir del 26/02/20 - 24/05/2020, cuando se tomaron varias medidas cautelares, como el uso de mascarillas, higiene con gel de alcohol y la reducción del flujo de personas en lugares públicos a través de cuarentena, por ejemplo. Debido a esto, la lucha por la movilización de recursos se ha vuelto aún más desafiante para las ONG.

En la red social Facebook, antes de la pandemia hubo 17.808 interacciones, de las cuales 1.552 estaban relacionadas con el objetivo 1 (comunicación); 978 al objetivo 2 (solidaridad) para atraer donaciones y voluntarios; el objetivo 3 (institucional), involucrar a la sociedad civil con la mayor cantidad de interacciones, 10.584 y finalmente el objetivo 4 (transparencia), totalizando 4.694 compromisos. 
Durante la pandemia los resultados fueron aproximados, pero hubo menos interacción de los usuarios, sólo 17,702. El objetivo 1 (comunicación) incrementó significativamente el número de interacciones, de 1.552 a 3.884, así como el objetivo 2 (solidaridad), que pasó de 978 a 1.848; el objetivo 3 (institucional) tuvo menos interacciones en este período, antes con 10.584, luego con 8.605. El objetivo 4 (transparencia) también estuvo menos comprometido antes de $4.694 \mathrm{y}$ durante de 3.365 .

Tabla 5

Engagement antes/durante COVID-19 en Facebook

\begin{tabular}{|c|c|c|c|c|c|c|c|c|c|c|c|c|}
\hline \multicolumn{10}{|c|}{ ENGAGEMENT ANTES DE LA PANDEMIA PROVOCADA POR EL COVID-19 (24/11/2019 a 25/02/2020) - FACEBOOK } \\
\hline & $\begin{array}{c}\text { ME } \\
\text { GUSTA }\end{array}$ & AMADO & TRISTE & GUAU & GRR & FUERZA & JAJA & $\begin{array}{c}\text { COMENT. } \\
\text { POSITIVO }\end{array}$ & $\begin{array}{c}\text { COMENT. } \\
\text { NEUTRO }\end{array}$ & $\begin{array}{c}\text { COMENT. } \\
\text { NEGATIVO }\end{array}$ & $\begin{array}{c}\text { COMP. } \\
\text { ENGAGEMENT }\end{array}$ \\
\hline OBJ 1 & 1.103 & 113 & 0 & 13 & 0 & 0 & 0 & 96 & 71 & 2 & 154 & 1.552 \\
\hline OBJ 2 & 715 & 54 & 1 & 0 & 0 & 0 & 1 & 25 & 4 & 0 & 178 & 978 \\
\hline OBJ 3 & 7.914 & 1.407 & 2 & 30 & 4 & 0 & 1 & 458 & 38 & 1 & 729 & 10.584 \\
\hline OBJ 4 & 3.754 & 317 & 3 & 17 & 4 & 0 & 0 & 190 & 14 & 1 & 3944 & 4.694 \\
\hline
\end{tabular}

\begin{tabular}{|c|c|c|c|c|c|c|c|c|c|c|c|c|}
\hline \multicolumn{13}{|c|}{ ENGAGEMENT DURANTE LA PANDEMIA PROVOCADA POR EL COVID-19 (26/02/2020 a 24/05/2020) - FACEBOOK } \\
\hline & $\begin{array}{c}\text { ME } \\
\text { GUSTA }\end{array}$ & AMADO & TRISTE & GUAU & GRR & FUERZA & JAJA & $\begin{array}{l}\text { COMENT. } \\
\text { POSITIVO }\end{array}$ & $\begin{array}{l}\text { COMENT. } \\
\text { NEUTRO }\end{array}$ & $\begin{array}{r}\text { COMENT. } \\
\text { NEGATIVO }\end{array}$ & COMP. & ENGAGEMENT \\
\hline OBJ 1 & 2.655 & 389 & 4 & 6 & 0 & 1 & 3 & 199 & 49 & 3 & 575 & 3.884 \\
\hline OBJ 2 & 1.058 & 76 & 8 & 5 & 0 & 2 & 0 & 14 & 29 & 0 & 656 & 1.848 \\
\hline OBJ 3 & 5.961 & 1.294 & 1 & 10 & 0 & 13 & 4 & 425 & 48 & 0 & 849 & 8.605 \\
\hline OBJ 4 & 2.511 & 342 & 3 & 9 & 1 & 5 & 0 & 127 & 17 & 0 & 350 & 3.365 \\
\hline
\end{tabular}

Fuente: elaboración propia.

En Instagram, el número total de interacciones antes de la pandemia fue de 7.805. El objetivo 1 obtuvo 624 interacciones y el objetivo 2 resultó en 643 compromisos. La meta institucional 3 representó el mayor compromiso con 5.786 interacciones y el objetivo 4, relacionado con la transparencia, reunió 752 interacciones.

Después del inicio de la pandemia, las interacciones de Instagram aumentaron considerablemente, de 7.805 casi se duplicaron a 14.636. De ellas, 2.991 interacciones estuvieron relacionadas con el objetivo $1 ; 1.886$ con respecto al objetivo 2; 6.686 para el objetivo 3 y 3.073 corresponden al objetivo 4 . Como se muestra en la siguiente tabla, todos los objetivos tuvieron un aumento en las interacciones en este período.

Tabla 6

Engagement antes/durante COVID-19 en Instagram

\begin{tabular}{|l|c|c|c|c|c|}
\hline \multicolumn{5}{|c|}{ ENGAGEMENT ANTES DE LA PANDEMIA PROVOCADA POR EL COVID-19 (24/11/2019 a 25/02/2020) - INSTAGRAM } \\
\hline & ME GUSTA & $\begin{array}{c}\text { COMENT. } \\
\text { POSITIVO }\end{array}$ & $\begin{array}{c}\text { COMENT. } \\
\text { NEUTRO }\end{array}$ & $\begin{array}{c}\text { COMENT. } \\
\text { NEGATIVO }\end{array}$ & ENGAGEMENT \\
\hline OBJETIVO 1 & 610 & 7 & 6 & 1 & 624 \\
\hline OBJETIVO 2 & 628 & 13 & 2 & 0 & 643 \\
\hline OBJETIVO 3 & 5.670 & 99 & 17 & 0 & 5.786 \\
\hline OBJETIVO 4 & 734 & 15 & 3 & 0 & 752 \\
\hline
\end{tabular}

\begin{tabular}{|l|c|c|c|c|c|}
\hline \multicolumn{5}{|c|}{ ENGAGEMENT DURANTE LA PANDEMIA PROVOCADA POR EL COVID-19 (26/02/2020 a 24/05/2020) - INSTAGRAM } \\
\hline & ME GUSTA & $\begin{array}{c}\text { COMENT. } \\
\text { POSITIVO }\end{array}$ & $\begin{array}{c}\text { COMENT. } \\
\text { NEUTRO }\end{array}$ & $\begin{array}{c}\text { COMENT. } \\
\text { NEGATIVO }\end{array}$ & ENGAGEMENT \\
\hline OBJETIVO 1 & 2.872 & 110 & 9 & 0 & 2.991 \\
\hline OBJETIVO 2 & 1.846 & 34 & 6 & 0 & 1.886 \\
\hline OBJETIVO 3 & 6.481 & 197 & 8 & 0 & 6.686 \\
\hline OBJETIVO 4 & 2.977 & 95 & 1 & 0 & 3.073 \\
\hline
\end{tabular}

Fuente: elaboración propia.

En Twitter, antes de la pandemia, no existían interacciones, independientemente del objetivo analizado. Por lo tanto, todas las interacciones tuvieron lugar cuando el COVID-19 ya estaba presente en Brasil. Con sólo 7 interacciones, 4 estaban relacionadas con el objetivo 3, y 3 con el objetivo 4 de mejorar la rendición de cuentas y la transparencia. 
Tabla 7

Engagement antes/durante COVID-19 en Twitter

\begin{tabular}{|l|c|c|c|c|c|c|}
\hline \multicolumn{6}{|c|}{ ENGAGEMENT ANTES DE LA PANDEMIA PROVOCADA POR EL COVID-19 (24/11/2019 a 25/02/2020) - TWITTER } \\
\hline & ME GUSTA & $\begin{array}{c}\text { COMENT. } \\
\text { POSITIVO }\end{array}$ & $\begin{array}{c}\text { COMENT. } \\
\text { NEUTRO }\end{array}$ & $\begin{array}{c}\text { COMENT. } \\
\text { NEGATIVO }\end{array}$ & COMP. & ENGAGEMENT \\
\hline OBJETIVO 1 & 0 & 0 & 0 & 0 & 0 & 0 \\
\hline OBJETIVO 2 & 0 & 0 & 0 & 0 & 0 & 0 \\
\hline OBJETIVO 3 & 0 & 0 & 0 & 0 & 0 & 0 \\
\hline OBJETIVO 4 & 0 & 0 & 0 & 0 & 0 & 0 \\
\hline
\end{tabular}

\begin{tabular}{|l|c|c|c|c|c|c|}
\hline \multicolumn{6}{|c|}{ ENGAGEMENT DURANTE LA PANDEMIA PROVOCADA POR EL COVID-19 (26/02/2020 a 24/05/2020) - TWITTER } \\
\hline & ME GUSTA & $\begin{array}{c}\text { COMENT. } \\
\text { POSITIVO }\end{array}$ & $\begin{array}{c}\text { COMENT. } \\
\text { NEUTRO }\end{array}$ & $\begin{array}{c}\text { COMENT. } \\
\text { NEGATIVO }\end{array}$ & COMP. & ENGAGEMENT \\
\hline OBJETIVO 1 & 0 & 0 & 0 & 0 & 0 & 0 \\
\hline OBJETIVO 2 & 0 & 0 & 0 & 0 & 0 & 0 \\
\hline OBJETIVO 3 & 3 & 0 & 0 & 0 & 1 & 4 \\
\hline OBJETIVO 4 & 2 & 0 & 0 & 0 & 1 & 3 \\
\hline
\end{tabular}

Fuente: elaboración propia.

En cuanto a la presencia en redes sociales por número de publicaciones, el resultado previo a la pandemia fue de 580 publicaciones, 119 de ellas relacionadas con el objetivo 1 de comunicación con los beneficiarios; 50 para recaudar donaciones y voluntarios; 352 involucrando a la sociedad civil y 59 con el objetivo de mejorar la rendición de cuentas y la transparencia. Durante la pandemia el número aumentó, el número total de publicaciones fue de 1.131; 344 abordar el objetivo 1 (comunicación); 122 objetivo 2 (solidario); 521 objetivo 3 (institucional) y 144 objetivo 4 (transparencia).

\section{Tabla 8}

Resumen de publicaciones antes y durante COVID-19

\begin{tabular}{|c|c|c|c|c|c|}
\hline \multicolumn{5}{|c|}{ PUBLICACIONES ANTES DE LA PANDEMIA PROVOCADA POR EL COVID-19 (24/11/2019 a 25/02/2020) } \\
\hline & REDE SOCIAL & OBJETIVO 1 & OBJETIVO 2 & OBJETIVO 3 & OBJETIVO 4 \\
\hline $\mathbf{1}$ & FACEBOOK & 84 & 29 & 184 & 41 \\
\hline $\mathbf{2}$ & INSTAGRAM & 23 & 20 & 150 & 18 \\
\hline $\mathbf{3}$ & TWITTER & 12 & 1 & 18 & 0 \\
\hline \multicolumn{2}{|c|}{ TOTAL GENERAL } & $\mathbf{1 1 9}$ & $\mathbf{5 0}$ & $\mathbf{3 5 2}$ & $\mathbf{5 9}$ \\
\hline
\end{tabular}

\begin{tabular}{|c|c|c|c|c|c|}
\hline \multicolumn{5}{|c|}{ PUBLICACIONES DURANTE LA PANDEMIA PROVOCADA POR EL COVID-19 (26/02/2020 a 24/05/2020) } \\
\hline & REDE SOCIAL & OBJETIVO 1 & OBJETIVO 2 & OBJETIVO 3 & OBJETIVO 4 \\
\hline $\mathbf{1}$ & FACEBOOK & 205 & 66 & 331 & 81 \\
\hline $\mathbf{2}$ & INSTAGRAM & 125 & 52 & 175 & 62 \\
\hline $\mathbf{3}$ & TWITTER & 14 & 4 & 15 & 1 \\
\hline & TOTAL GENERAL & $\mathbf{3 4 4}$ & $\mathbf{1 2 2}$ & $\mathbf{5 2 1}$ & $\mathbf{1 4 4}$ \\
\hline
\end{tabular}

Fuente: elaboración propia.

\subsection{Análisis y discusión}

Al analizar la presencia de ONG en el entorno online, se consideraron tres redes sociales: Facebook, Instagram y Twitter. Se identificó que la red social más utilizada es Facebook, en la que se produjeron la mayoría de interacciones y están presentes las 14 organizaciones analizadas. La segunda red social más utilizada es Instagram, donde 13 ONG tienen una página activa. El uso de Instagram aumentó considerablemente en el período en que comenzó el COVID-19 en Brasil, posiblemente debido a de que muchos artistas, empresas y organizaciones utilizaron la función "en vivo" de Instagram para comunicarse con los involucrados y recaudar fondos. Por tanto, las organizaciones del tercer sector vieron la necesidad de ser más activas en 
esta red social. En el caso de Twitter, se observó que sólo fue utilizada por una ONG de las 14 analizadas.

La mayoría de las veces, el contenido que se publicó en Facebook fue replicado en otras redes sociales. Por lo tanto, se vio que muchas organizaciones sin fines de lucro aún no utilizan todo el potencial de su presencia en Internet. En cuanto a las publicaciones, se pudo notar que algunas ONG tienen mayor planificación en cuanto a frecuencia de publicaciones y calidad de contenido que otras, que en un día publican más de cinco veces y en los próximos días no realizan publicación alguna. Por ello, se considera necesaria una buena planificación de comunicación estratégica en las ONG. Según Barros (2013) muchas organizaciones no pueden permitirse contratar personas calificadas para preparar y aplicar tales estrategias. El argumento más común es la falta de recursos económicos para mantener un departamento específico dedicado al trabajo de comunicación (Román-San Miguel y Díaz, 2019). Sin embargo, algunas ONG prefieren no invertir en un profesional para cuidar sus medios digitales porque aún no entienden la eficiencia e impacto que esto puede traer a sus resultados.

Paulatinamente el escenario ha ido cambiando. El COVID-19 ha tenido un impacto en las estructuras y procesos de muchas organizaciones que comenzaron a reconocer el valor de las redes sociales en su comunicación, especialmente por su gran potencial para comunicarse con muchas personas al instante y a bajo costo. La necesidad de aislamiento social y cierre de los lugares de trabajo, para evitar la propagación del virus, trajo otro problema, la crisis económica. Muchas personas perdieron su empleo o tuvieron jornada reducida, según datos del Ministerio de Economía brasilero entre marzo y abril se registró un aumento del $31 \%$ en la solicitud del seguro de desempleo, respecto al mismo período del año pasado. Sin embargo, la filantropía individual e institucional ha sido receptiva, comprometida y ágil. La disposición para participar en una comunicación dialógica con el público se hizo evidente en estos momentos de crisis.

Con relación a las estrategias de comunicación más utilizadas por las ONG en las redes sociales Facebook, Twitter e Instagram en la ciudad de Blumenau, se pudo observar que tales organizaciones demostraron ser aún más imprescindibles durante la pandemia, ya sea para comunicar consejos para aplanar la curva de contaminación y buenas prácticas de distanciamiento social, informar cambios operacionales y nuevas medidas de asistencia para comunidades vulnerables de contagio, así como anunciar la ejecución de proyectos sociales que buscaron atraer donaciones a familias impactadas por la crisis económica.

El objetivo de comunicación más utilizado por las organizaciones fue involucrar a la sociedad civil (52\%). Este tipo de comunicación es institucional y busca establecer una relación activa con la sociedad. Los resultados demuestran que movilizar la participación ciudadana digital aprovechando las redes sociales juega un papel significativo para las organizaciones sin fines de lucro. Compartir información y organizar la asistencia humanitaria puede canalizarse hacia un apoyo constructivo. La sociedad civil resulta importante en garantizar que determinados recursos lleguen a su destino.

El segundo objetivo más aplicado fue comunicarse con los beneficiarios (25\%). En este caso, las publicaciones se centraron en consejos y actividades, que fue muy utilizada durante la pandemia. Durante la investigación fue posible observar algunos cambios en la comunicación de las ONG en sus redes sociales durante la pandemia. En Facebook, las interacciones con publicaciones de carácter organizativo y solidario se incrementaron considerablemente, ya que, 
al igual que otras empresas, las organizaciones no gubernamentales también tuvieron que paralizar actividades in loco durante un tiempo determinado, por ello, organizaciones como APAE - Associação de Padres y Amigos de los Excepcionales y ABADA - Asociación Blumenauense de Amigos de Discapacitados Auditivos publicaron actividades y consejos para que sus alumnos las realizaran en casa y siguieran recibiendo asistencia, aunque sea a distancia (Figura 1).

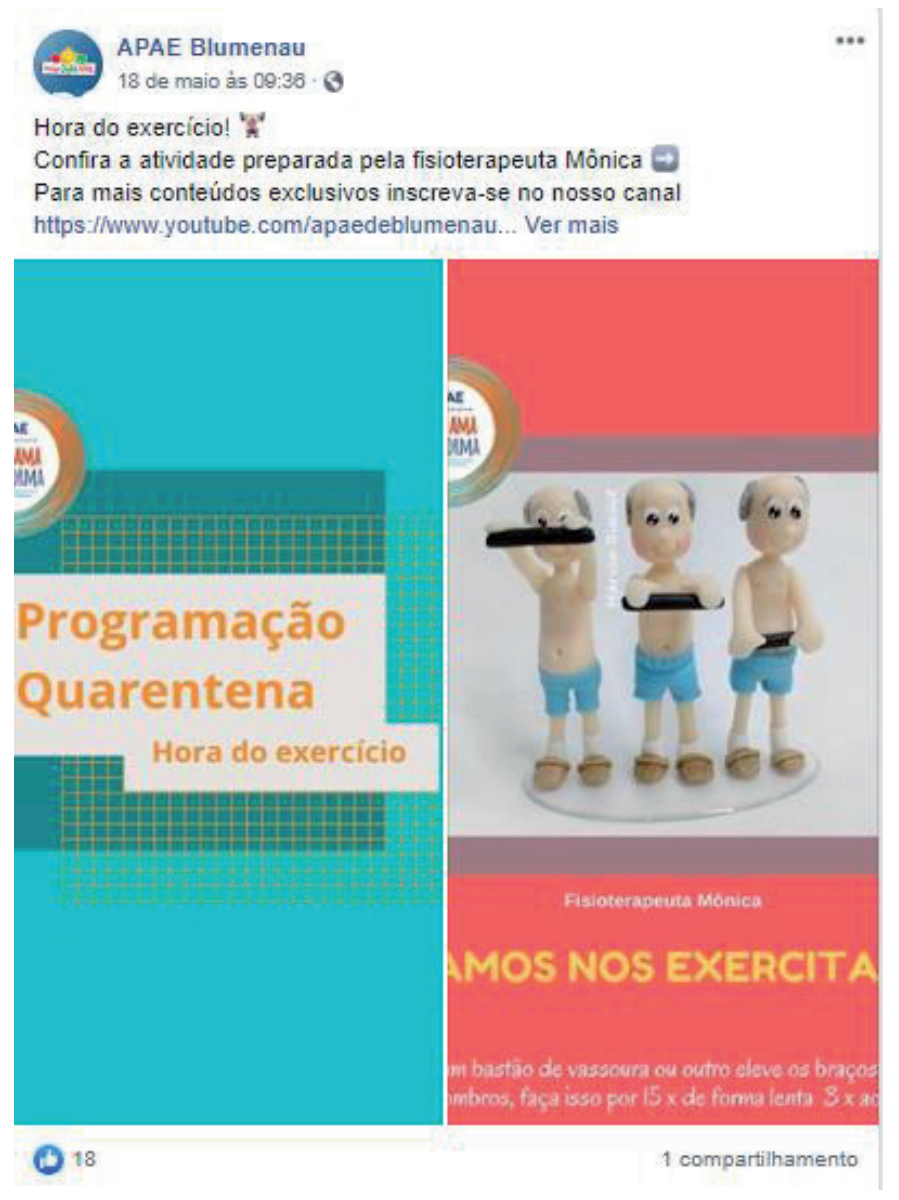

Figura 1. Publicación en Facebook (APAE Blumenau)

Fuente: Facebook/Elaboración propia.

En tercera posición, el objetivo que representó el 12\% de las publicaciones está relacionado con la responsabilidad y la transparencia. Tales publicaciones, de manera general, divulgaron los valores recogidos en sus acciones sociales, así como las donaciones recibidas de alguna institución. Por otra parte, si bien uno de los principales propósitos de comunicación de estas organizaciones es atraer voluntarios y donaciones, este fue el objetivo menos utilizado por ellas $(9,2 \%)$. Esto posiblemente ocurrió porque, aunque no directamente, todos los demás objetivos contribuyeron de alguna manera al mismo propósito, la obtención de recursos.

En medio de la pandemia, las organizaciones buscaron simplificar los procesos de recolección de donaciones y los donantes individuales se asociaron con sus pares para realizar donativos considerables con menos burocracia. A través de Internet y las redes sociales, muchas instituciones crearon soluciones para recolectar las donaciones realizadas durante el aislamiento. Además, con eventos cancelados, muchos artistas transmitieron sus programas en 
línea, llamados "cuarentena en vivo" y los transformaron en un instrumento de recaudación de fondos para las ONG (Figura 2).

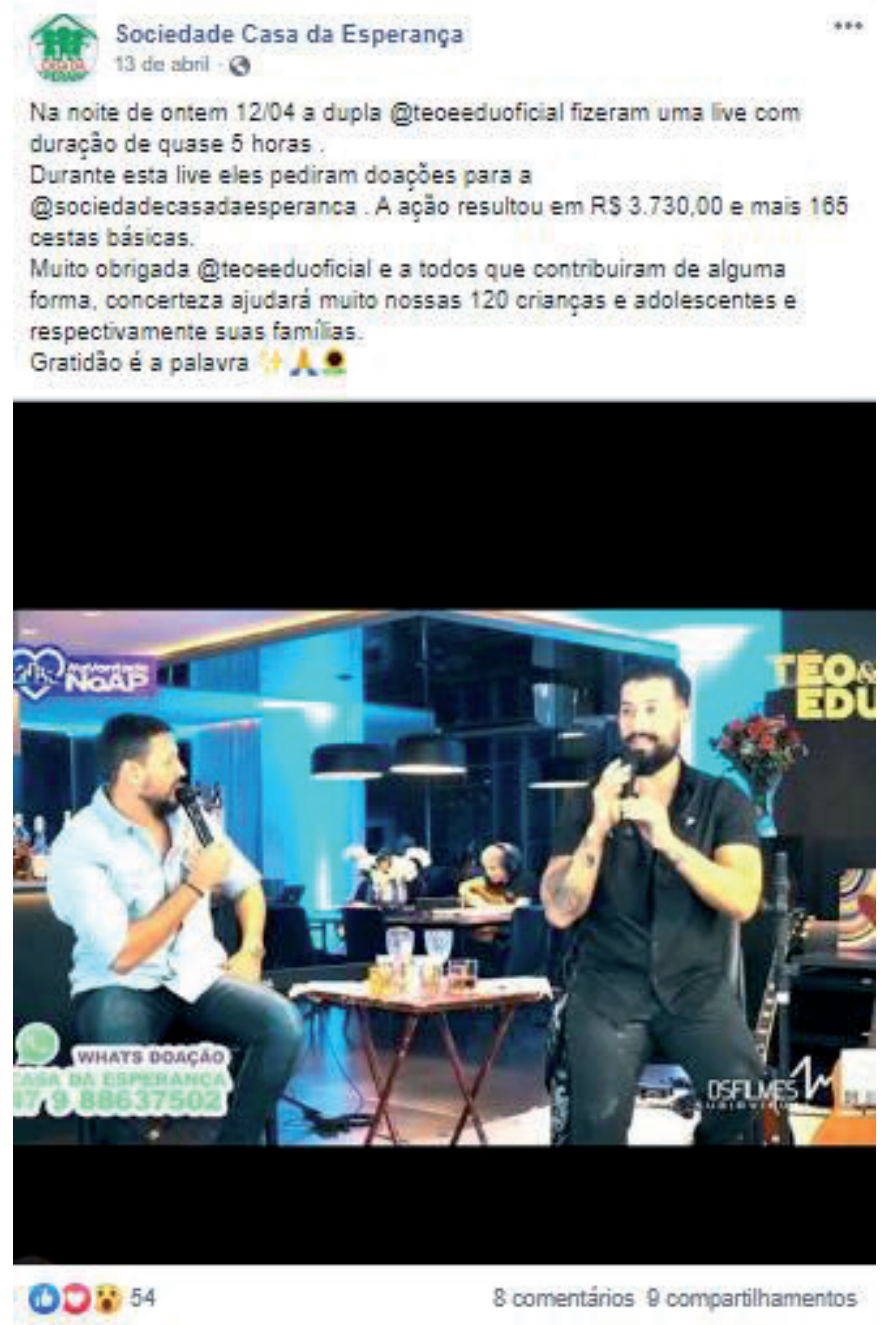

Figura 2. Publicación en Facebook (Sociedade Casa da Esperança)

Fuente: Facebook/Elaboración propia.

Al analizar el nivel de engagement de los usuarios en las redes sociales de las ONG estudiadas, se observó que el objetivo que obtuvo mayor engagement en todas las redes sociales fue el 3, involucrar a la sociedad civil, con un $54 \%$ de interacciones. En segundo lugar, el objetivo 4 , de mejorar la rendición de cuentas y la transparencia, fue del 20\%; luego el objetivo 1 de comunicarse con los beneficiarios, con un $15 \%$ de interacciones y finalmente el objetivo 2 , de atraer donaciones y voluntarios, con un $9,2 \%$ de compromisos. Sin embargo, como ya se mencionó, es posible que haya dado el mismo resultado debido a que las ONG no personalizan el contenido para cada medio, sino que replican las publicaciones realizadas en Facebook.

Las reacciones son la forma más rápida y sencilla para que los usuarios demuestren lo que sienten sobre el contenido que reciben. En el presente estudio, las organizaciones recibieron 52.444 reacciones, principalmente positivas. Incluso aquellas consideradas negativas, como triste y grr, en el contexto analizado también son positivas porque demuestran empatía con el tema de la publicación. Como comenta Vivek (2014) esto representa el nivel de entusiasmo en 
la implicación del usuario con la organización, ya que la implicación afectiva se manifiesta a través de interacciones positivas. En el engagement de todas las publicaciones analizadas, independientemente de la red social, hubo pocos comentarios negativos, lo que representa una buena relación con los usuarios.

Otra situación que llamó la atención fue la presencia activa y el aumento del engagement en la plataforma Instagram, que fue aproximadamente del 100\%, en comparación con el período anterior de la pandemia. En general, tanto la presencia como la participación de los usuarios en las páginas de las ONG aumentaron durante el período de la pandemia. El número de publicaciones aumentó en un $51,28 \%$ y el engagement, contado a través de reacciones, comentarios y compartidos, también aumentó en un 79,18\% respecto al período anterior, con 25.613 interacciones antes y 32.345. Estos resultados reafirman el papel destacado que desempeñan actualmente las redes sociales, especialmente durante la pandemia de COVID-19.

\section{CONCLUSIONES}

En términos generales se puede concluir que la red social con mayor presencia es Facebook, seguida por Instagram y por último Twitter. Los resultados destacaron la importancia de la presencia de las ONG en las redes sociales, especialmente durante la pandemia, debido a que estas se convirtieron en un amplificador de la labor de tales entidades, como vía para encontrar apoyo, sensibilizar a la sociedad civil e incorporar a nuevos voluntarios.

La estrategia más utilizada fue involucrar a la sociedad civil, fundamentalmente durante el segundo período analizado. Por lo general, el contenido divulgado no aparentó proceder de una planificación con cronograma de publicaciones, pues muchos de los contenidos vehiculados por las ONG se realizaban de modo aleatorio. Sobre este asunto, cabe resaltar la importancia de la planificación estratégica, tema que ha recibido especial atención en la literatura científica reciente (Esparcia et al., 2017; Arroyo-Almaraz y Calle Mendoza, 2018; González et al., 2018; Román-San-Miguel y Díaz, 2019). Se debe tener claro el propósito de los contenidos compartidos, así como cuál canal es el más conveniente para hacerlo, puesto que no todos los materiales sirven en todas las redes sociales, en tanto no tienen el mismo impacto. Con relación al engagement de los usuarios, las interacciones más recurrentes fueron por medio de reacciones y publicaciones compartidas, ya sea al marcar amigos en los comentarios o usando la opción de compartir publicación.

Con base en los resultados de este estudio, se sugiere que las investigaciones futuras examinen temas relacionados con las implicaciones de la comunicación digital de las organizaciones sin fines de lucro en Blumenau-SC y comprender cuáles son sus desafíos y dificultades.

\section{Referencias}

Arora, A., Bansal, S., Kandpal, C., Aswani, R., \& Dwivedi, Y. (2019). Measuring social media influencer index- insights from Facebook, Twitter and Instagram. Journal of Retailing and Consumer Services, 49(3), 86-101.

Arroyo-Almaraz, I., \& Calle Mendoza, S. (2018). Los community managers de las ONGD. Estudio de percepciones y usos de las redes sociales. Revista ICONO14 Revista Científica De Comunicación y Tecnologías Emergentes, 16(2), 121-142.

Bardin, L. (2006). Análise de conteúdo. Lisboa: L. de A. Rego \& A. Pinheiro.

Barros, R. (2013). Marketing social aplicado às ONGs. https://bit.ly/3rPVbLz 
Campbell, D. \& Lambright, K. (2019). Are You Out There? Internet Presence of Nonprofit Human Service Organizations. Sage, 1-16. https://doi.org/10.1177/0899764019852673

Chrysochou, P. \& Malciute, J. (2013). Customer Brand Engagement on Online Social Media Platforms: A Conceptual Model and Empirical. https://bit.ly/3eHUAYA

Deschamps, R. \& McNutt, K. (2014). Third sector and social media. Canadian journal of nonprofit and social economy research, 5(2), 29-46.

Dolnicar, S. \& Lazarevski, K. (2009). Marketing in non-profit organization: an international perspective. International Marketing Review, 26(1), 275-291.

Esparcia, A. C., Kunsch, M. M. K., \& Haswani, M. F. (2017). Prácticas comunicativas y perspectivas para el cambio social en las organizaciones no gubernamentales (ONGs), en España y Brasil. ORGANICOM, 14(26), 147-166.

Fitzgerald, E. (2019). Branding and marketing for an environmental nonprofit organization: revitalizing a brand in Southern Illinois. https://bit.ly/3eGK3wB

Gabriel, M. (2017) Marketing na era digital: conceitos, plataformas e estratégias. São Paulo: Novatec.

González, E. R., Luis, H. G., \& Esteban, P. P. (2018). Los medios en la gestión de la comunicación de las ONG en tiempos de crisis: oportunidades para el cambio social. Observatorio (OBS), 12(3), 83105.

Greenberg, J. \& Macaulay, M. (2009). NPO 2.0? Exploring the Web Presence of Environmental Nonprofit Organizations in Canada, Global Media Journal: Canadian Edition, 63-88.

Higgins, E. \& Scholer, A. (2009). Engaging the consumer: The science and art of the value creation process. Journal of Consumer Psychology, 19(2), 100-114.

Instituto Brasileiro de Geografia e Estatística (2019). As fundações privadas e associações sem fins lucrativos no Brasil: 2016. Estudos e Pesquisas, 32. https://bit.ly/2NrqQnC

Keegan, B. \& Rowley, J. (2017) Evaluation and decision making in social media marketing. Management Decision, 55(1), 15-31.

Kotler, P. \& Lee. N. (2008). Social marketing: influencing behaviors for good. Los Angeles: Sage.

Kotler, P. \& Levy, S.J. (1969). Broadening the Concept of Marketing. Journal of Marketing, 33(1), 10-15.

Lovejoy, K. \& Saxton, G. (2012) Information, community, and action: how nonprofit organizations use social media. Journal of Computer-Mediated Communication, 17(3), 337-353.

Machado, L. \& Almeida, M. (2015) O papel da comunicação nas organizações do terceiro setor: um estudo de caso do Gacc de Sergipe. Temática, 1(6), 101-114.

Mattar, F.N. (2001). Pesquisa de Marketing. São Paulo: Atlas.

Mello, J., Pereira, A. C. R., \& Andrade, P. G. (2019). Afinal, o que os dados mostram sobre a atuação das ONGs? Análise de transferências federais e projetos executados pelas organizações da sociedade civil no Brasil. IPEA. https://bit.ly/2OTBCTS

Morales, D. (2016). Una aproximación al marketing social. Pensamiento \& Gestión, 41, 7-12.

Moreira, J. (2014). A comunicação comunitária e as instituições sem fins lucrativos: O caso da Sociedade Beneficente Mão Amiga. https://bit.ly/3tncd3P

Peruzzo, C. (2013). Movimentos sociais, redes virtuais e mídia alternativa no junho em que "O Gigante Acordou". Revista Matrizes (ECA-USP), 7(2), 73-93.

Pope, J. et al. (2009). Developing a marketing strategy for nonprofit organizations: an exploratory study. Journal of nonprofit \& public sector marketing, 21(2), 184-201.

Rocha, E. (2018). A influência das experiências memoráveis no engajamento dos turistas na mídia social virtual: perspectivas a partir dos parques temáticos Beto Carrero World (SC, Brasil) e Port Aventura World (TGN, Espanha). https://bit.ly/2PZ38Qk

Román-San Miguel, A. \& Díaz, J. (2019). Communication and advertising in NGDOs: Present and future. 
IROCAMM, 2(1), 95-101.

Silva, V. (2018). As mídias sociais e sua contribuição na comunicação de ONGs. Revista Especialize On-line Ipog, 16(1), 1-14.

Tian, F., Labban, A., Shearer, R., \& Gai, Q. (2019). The Impact of Social Media Activity on Nonprofit Donations in China. VOLUNTAS: International Journal of Voluntary and Nonprofit Organizations, 1-10. https://doi.org/10.1007/s11266-019-00168-7

Vieira, V. A., de Almeida, M. I. S., Agnihotri, R., \& Arunachalam, S. (2019). In pursuit of an effective B2B digital marketing strategy in an emerging market. Journal of the Academy of Marketing Science, 47(6), 1085-1108.

Vivek, S. (2014). A generalized multidimensional scale for measuring customer engagement. Journal of Marketing Theory and Practice, 22(4), 401-420.

\section{Semblanza de los autores}

Fabricia Durieux Zucco: Es doctora en Administración por la Universidade Nove de Julho, São Paulo, Brasil, tiene un máster en Administración y es licenciada en Comunicación Social por la Universidade Regional de Blumenau, Blumenau, Santa Catarina, Brasil. Es profesora del Curso de Postgrado en Turismo y Hostelería de la Universidad de Vale do Itajaí, Balneário Camboriú, Santa Catarina, Brasil y del Programa de Posgrado en Administración de la Universidad Regional de Blumenau, Blumenau, Santa Catarina, Brasil.

Juliana Machado: Licenciada en Comunicación Social (publicidad y propaganda) por la Universidad Regional de Blumenau, Blumenau, Santa Catarina, Brasil.

Cynthia Morgana Boos de Quadros: Es doctora en Desarrollo Regional por la Universidad Regional de Blumenau, Blumenau, Santa Catarina, Brasil y tiene un máster en Ciencias del Lenguaje por la Universidad del Sur de Santa Catarina. Es profesora del Programa de Posgrado en Administración de la Universidad Regional de Blumenau y enseña en cursos de pregrado y de especialización en las áreas de Comunicación, Administración y Marketing. Tiene experiencia académica y de mercado en las áreas de investigación de marketing, opinión pública, estudios de consumo y comunicación regional.

Thamires Foletto Fiuza: Tiene un máster en turismo y hostelería por la Universidad de Vale do Itajaí, Balneário Camboriú, Santa Catarina, Brasil y una licenciatura en turismo por la Universidad Federal de Río Grande, Santa Vitória do Palmar, Rio Grande del Sur, Brasil. 